\title{
Making germanes relevant in cross-coupling
}

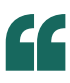

We wondered

if germanes

might prove

to be a

more useful

transmetal-

ation agent

with a different

metal catalyst
Cross-coupling is a catch-all term for reactions in which two prefunctionalized fragments - most often one halide or pseudohalide and one organometallic or organometalloid - are joined with the aid of a Palladium-catalysed cross-coupling reactions in particular have revolutionized synthetic chemistry. These reactions are now such a wellestablished synthetic tool that it is difficult to imagine how we would synthesize many of the molecules of the modern world without them. Now, Franziska Schoenebeck and co-workers from RWTH Aachen University have added a new orthogonal cross-coupling reaction to the toolkit: gold-catalysed crosscouplings of aryl germanes with diazoarenes.

"Despite the many metal catalysts and transformations that have been described, we still largely rely on the few transmetalation reagents that were originally discovered in the context of palladium-catalysed cross-coupling," says Schoenebeck. It is perhaps surprising that while silanes and stannanes have been successfully used in cross-couplings, germanes have rarely proved useful. But while there have been a few reports on their use in Pd-catalysed cross-couplings, researchers have always concluded that germanes are less reactive than other coupling metal catalyst to form a new bond. partners. "We wondered if germanes might prove to be a more useful transmetalation agent with a different metal catalyst," Schoenebeck continues.

The work began with a detailed experimental and computational study of Pd-based chemistry. This revealed a fundamental problem: the transmetalation reaction at typical Pd(II) intermediates was highly endergonic - there was no driving force for the reaction - and the transition state was prohibitively high. "We did find, however, that palladium nanoparticles, unlike their homogeneous catalyst counterparts, catalysed cross-coupling of germanes and did so in preference to silanes, boronic acids or boronic esters," says Schoenebeck. This observation as well as detailed computational studies led to the conclusion that germanes react preferentially in electrophilic aromatic substitution-type processes. "This explains why an electrophilic metal catalyst such as a cationic palladium nanoparticle was effective and, in turn, led us to try coupling using a gold catalyst," she continues.

Where coupling of germanes was poor or slow with typical homogeneous palladium catalysis, they become the preferred coupling partner under gold catalysis. And, although gold catalysis also enables the coupling of unfunctionalized arenes using $\mathrm{C}-\mathrm{H}$ activation,
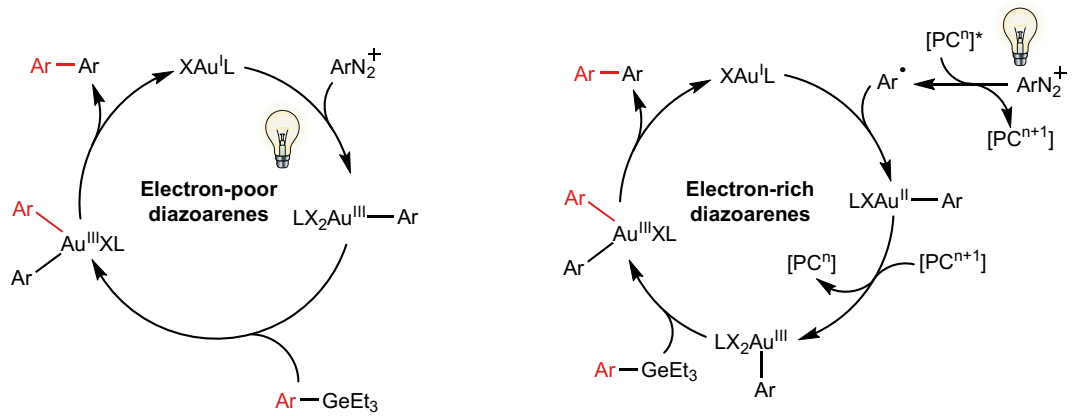

Credit: Stephen G. Davey/Springer Nature Limited the team pursued coupling with diazoarenes (activated by irradiation with blue light), meaning that they could avoid the need to include a stoichiometric oxidant in their reaction. Taking all these elements together, Schoenebeck and co-workers were able to develop a coupling reaction entirely orthogonal to the current toolbox.

Electron-poor diazoarenes were easily coupled under visible light with remarkable tolerance to other functionalities. In order to apply electron-rich diazoarenes, an additional photocatalyst was found to be an essential additive to the reaction. This photocatalyst helps to avoid decomposition of the diazoarene by a competing pathway that was computationally found to be thermodynamically favoured for these substrates. Thus, depending on the electronic properties of the diazoarene, the reaction proceeds by two fundamentally different mechanisms.

The orthogonality of these reactions to the standard toolbox of coupling reactions is particularly noteworthy. "Being able to couple germanes fully selectively in the presence of silanes, BPin and halogens means that we can use our gold-catalysed process to react first with a germane and then harness more standard $\mathrm{Pd}, \mathrm{Cu}$ or other established chemistry to diversify other sites. In this way we're able to quickly generate libraries of compounds in a selective building block approach," says Schoenebeck.

Stephen G. Davey

ORIGINAL ARTICLE Sherborne, G. J. et al. Modular and selective arylation of aryl germanes ( $\mathrm{C}-\mathrm{GeEt}_{3}$ ) over $\mathrm{C}-\mathrm{Bpin}, \mathrm{C}-\mathrm{SiR}_{3}$ and halogens enabled by light-activated gold catalysis. Angew. Chem. Int. Ed. https://doi.org/10.1002/anie. 202005066 (2020) RELATED ARTICLE Fricke, C. et al. Gold-catalyzed C-H functionalization with aryl germanes. ACS Catal. 9, 9231-9236 (2019) 